\title{
ANALYTIC SOLUTION FOR THE REDUCED CROSS-SECTION AND ITS DERIVATIVES AT LOW $x$ BASED ON GLUON AND STRUCTURE FUNCTION EXPONENTS
}

\author{
G.R. Boroun \\ Physics Department, Razi University, Kermanshah 67149, Iran \\ E-mail: boroun@razi.ac.ir, grboroun@gmail.com
}

Received 29 December 2007; revised 2 March 2008; accepted 9 June 2008

\begin{abstract}
Analytic solutions for the reduced cross-section and its derivatives with respect to $\ln y$ are presented at the low- $x$ limit. The DGLAP evolution equations for singlet and gluon structure function based on Regge-like behaviour of the gluon distribution and the structure function at this limit are solved. We calculated numerically and compared our results with the HERA experiment H1 data at small $x$. All results can be consistently described within the framework of perturbative QCD.
\end{abstract}

Keywords: reduced cross-section, DGLAP evolution equation, small- $x$ physics, Regge-like behaviour

PACS: $13.60 . \mathrm{Hb}, 11.55 . \mathrm{Jy}$

\section{Introduction}

Deep-inelastic lepton-nucleon scattering (DIS) has been pivotal in the development of the understanding of strong interaction dynamics. Also, at small values of the Bjorken scaling variable $x$ it is interesting for understanding the inner structure of hadrons. In the one-photon exchange approximation the neutral current double differential cross-section, $\mathrm{d}^{2} \sigma /\left(\mathrm{d} x \mathrm{~d} Q^{2}\right)$, is given by the expression

$$
\frac{\mathrm{d}^{2} \sigma}{\mathrm{d} x \mathrm{~d} Q^{2}}=\frac{2 \pi \alpha^{2} Y_{+}}{Q^{4} x} \sigma_{\mathrm{r}},
$$

where the reduced cross-section is defined as

$$
\sigma_{\mathrm{r}} \equiv F_{2}\left(x, Q^{2}\right)-\frac{y^{2}}{Y_{+}} F_{\mathrm{L}}\left(x, Q^{2}\right),
$$

with $Y_{+}=1+(1-y)^{2}$. Here $Q^{2}$ is the squared four-momentum transfer, $x$ denotes the Bjorken scaling variable, $y=Q^{2} /(s x)$ is the inelasticity, with $s$ the electron-proton centre of mass energy squared, and $\alpha$ is the fine structure constant [1-5].

The reduced cross-section depends on the two independent structure functions $F_{2}\left(x, Q^{2}\right)$ and $F_{\mathrm{L}}\left(x, Q^{2}\right)$. At low $x$, the structure functions' behaviour is well understood in terms of Regge-like behaviour [6]. Recent studies $[7,8]$ have shown that it is possible to use Regge theory for the Dokshitzer-Gribov-Lipatov-Altarelli-
Parisi (DGLAP) [9] evolution. Inserting this behaviour into the parton-model calculation of the structure functions gives us the small $x$ behaviour $F_{i=\mathrm{S}, \mathrm{g}} \sim x^{\lambda_{i=\mathrm{S}, \mathrm{g}}}$ as $x \rightarrow 0$, where $\lambda_{\mathrm{S}}$ and $\lambda_{\mathrm{g}}$ are the gluon and structure function exponents. Our goal in this work is to present an approximate analytical solution for the reduced crosssection and its derivatives. In order to do this, the DGLAP evolution equations are calculated neglecting the quark distribution. The approach, using the Regge and the Regge-like behaviour for singlet and gluon distribution respectively, has been applied in this paper. The formulation of the problem in next-to-leading order DGLAP (NLO-DGLAP) evolution equations for the calculation of the reduced cross-section and its derivatives through $\lambda_{\mathrm{g}}$ and $\lambda_{\mathrm{S}}$ exponents and numerical results are given in Section 2. Finally, Section 3 is devoted to calculation and results.

\section{Formalism}

In perturbative QCD, the longitudinal structure function $F_{\mathrm{L}}\left(x, Q^{2}\right)$ is proportional to $\alpha_{s}$. At low $x$ we use the fact that the non-singlet contribution $F_{2}^{\mathrm{NS}}$ can be ignored safely. Now we can write the longitudinal 
structure function by an integral over the quark and gluon distributions as [1,4]

$$
\begin{aligned}
F_{\mathrm{L}}\left(x, Q^{2}\right)= & \int_{x}^{1} \frac{\mathrm{d} y}{y} K_{\mathrm{q}}\left(\frac{x}{y}, Q^{2}\right) F_{2}\left(y, Q^{2}\right) \\
& +\int_{x}^{1} \frac{\mathrm{d} y}{y} K_{\mathrm{G}}\left(\frac{x}{y}, Q^{2}\right) G\left(y, Q^{2}\right)
\end{aligned}
$$

with

$$
F_{2}=x \sum_{i=1}^{n_{\mathrm{f}}} e_{i}^{2}[q(x)+\bar{q}(x)],
$$

where the structure function is given by a sum of quark and anti-quark momentum distribution functions. The gluon momentum density is given by $G\left(x, Q^{2}\right)=$ $x g\left(x, Q^{2}\right)$. Here $e_{i}$ are the quark charges and $n_{\mathrm{f}}=4$ the number of flavours, and the kernels are

$$
K_{\mathrm{q}}\left(x, Q^{2}\right)=\frac{\alpha_{s}}{4 \pi} 4 C_{F} x^{2}
$$

and

$$
K_{\mathrm{G}}\left(x, Q^{2}\right)=\frac{\alpha_{s}}{4 \pi}\left[8 x^{2}(1-x)\right] \sum_{i=1}^{n_{f}} e_{i}^{2},
$$

where $C_{F}$ is the colour Casimir operator. With substituting the splitting functions, $F_{\mathrm{L}}$ is obtained:

$$
\begin{aligned}
F_{\mathrm{L}}\left(x, Q^{2}\right)= & \frac{4 \alpha_{s}}{3 \pi} \int_{x}^{1} \frac{\mathrm{d} y}{y}\left(\frac{x}{y}\right)^{2} F_{2}\left(y, Q^{2}\right) \\
& +\frac{20 \alpha_{s}}{9 \pi} \int_{x}^{1} \frac{\mathrm{d} y}{y}\left(\frac{x}{y}\right)^{2}\left(1-\frac{x}{y}\right) G\left(y, Q^{2}\right) .
\end{aligned}
$$

The Regge-like behaviour of the gluon distribution function and the structure function is that the functions increase as $x$ decreases. This behaviour at small $x$ corresponds to a rising longitudinal structure function. Now let us use this behaviour, as the $x$ dependence of this at low $x$ is consistent with a power law for fixed $Q^{2}$ [10-15], as can be shown:

$$
\frac{\mathrm{d} F_{2}\left(x, Q^{2}\right)}{\mathrm{d} \ln x}=-\lambda_{\mathrm{S}} F_{2}\left(x, Q^{2}\right)
$$

and

$$
\frac{\mathrm{d} G\left(x, Q^{2}\right)}{\mathrm{d} \ln x}=-\lambda_{\mathrm{g}} G\left(x, Q^{2}\right),
$$

where $\lambda_{\mathrm{S}}$ and $\lambda_{\mathrm{g}}$ are the respective exponents. These functions are determined by the form $f_{i=F_{2}, G}=$
$C_{i=F_{2}, G} x^{-\lambda_{\mathrm{s}, \mathrm{g}}}$ where the coefficients $C$ are approximately independent of $Q^{2}$ with a constant mean value [16]. In a series of papers $[16,17]$ we have seen, $\lambda_{S, g}$ rises approximately linearly with $\ln Q^{2}$. This dependence can been represented as $\lambda_{\mathrm{S}, \mathrm{g}}=b_{\mathrm{S}, \mathrm{g}} \ln \left(Q^{2} / \Lambda^{2}\right)$. The coefficients $b_{\mathrm{S}, \mathrm{g}}$ are constant [16]. We are considering the similar scale $Q^{2} / Q_{0}^{2}$, so we can write the variation of $F_{2}\left(x, Q^{2}\right)$ and $G\left(x, Q^{2}\right)$ through the same functions. To begin with, the evolution of $\partial F_{2}\left(x, Q^{2}\right) / \partial \ln Q^{2}$ and $\partial G\left(x, Q^{2}\right) / \partial \ln Q^{2}$ at fixed $x$ values is obtained over all $Q^{2}$ values, as we have found:

$$
\frac{\partial F_{2}\left(x, Q^{2}\right)}{\partial \ln Q^{2}}=-b_{\mathrm{S}} \ln x F_{2}\left(x, Q^{2}\right)
$$

and

$$
\frac{\partial G\left(x, Q^{2}\right)}{\partial \ln Q^{2}}=-b_{\mathrm{g}} \ln x G\left(x, Q^{2}\right) .
$$

Therefore the gluon distribution and the structure function evolutions from the initial conditions are found as

$$
F_{2}\left(x, Q^{2}\right)=F_{2}\left(x, Q_{0}^{2}\right)\left(\frac{Q_{0}^{2}}{Q^{2}}\right)^{b_{s} \ln x}
$$

and

$$
G\left(x, Q^{2}\right)=G\left(x, Q_{0}^{2}\right)\left(\frac{Q_{0}^{2}}{Q^{2}}\right)^{b_{\mathrm{g}} \ln x} .
$$

On the basis of the DGLAP evolution equations it is known that the structure function and the gluon distribution function exponents can be evaluated. This fact makes it possible to relate the gluon and the structure function exponents to the initial exponents as has been suggested previously [16], i. e.

$$
\begin{aligned}
& \ln \frac{\lambda_{\mathrm{g}_{0}}}{\lambda_{\mathrm{g}}-x^{\lambda_{\mathrm{g}}} \int_{t_{0}}^{t} x^{-\lambda_{\mathrm{g}}}\left(\frac{3 \alpha}{\pi}-\frac{61 \alpha^{2}}{9 \pi^{2}}\right) \mathrm{d} t}= \\
& \int_{t_{0}}^{t}\left(\frac{3 \alpha}{\pi}-\frac{61 \alpha^{2}}{9 \pi^{2}}\right) \frac{1-x^{\lambda_{\mathrm{g}}}}{\lambda_{\mathrm{g}}} \mathrm{d} t,
\end{aligned}
$$

for the gluon distribution exponent evolution and

$$
\begin{aligned}
& \lambda_{\mathrm{S}} F_{2}(x, t)-\lambda_{\mathrm{S}_{0}} F_{2}\left(x, t_{0}\right)= \\
& \frac{0.555}{\pi} \int_{t_{0}}^{t} \alpha_{s} G(x, t)\left[\frac{2 \lambda_{\mathrm{g}}}{3+\lambda_{\mathrm{g}}}\left(1-x^{3+\lambda_{\mathrm{g}}}\right)\right. \\
& \quad+\frac{\lambda_{\mathrm{g}}}{1+\lambda_{\mathrm{g}}}\left(1-x^{1+\lambda_{\mathrm{g}}}\right)-\frac{2 \lambda_{\mathrm{g}}}{2+\lambda_{\mathrm{g}}}\left(1-x^{2+\lambda_{\mathrm{g}}}\right)+
\end{aligned}
$$




$$
\begin{aligned}
& \left.+2 x^{3+\lambda_{\mathrm{g}}}+x^{1+\lambda_{\mathrm{g}}}-2 x^{2+\lambda_{\mathrm{g}}}\right] \mathrm{d} t \\
& +\frac{1.852}{\pi^{2}} \int_{t_{0}}^{t} \alpha_{s}^{2} G(x, t) \mathrm{d} t,
\end{aligned}
$$

for the singlet structure function exponent evolution. The initial conditions are

$$
\lambda_{\mathrm{S}_{0}}=\frac{\partial \ln F_{2}\left(x, t_{0}\right)}{\partial \ln x^{-1}}
$$

and

$$
\lambda_{\mathrm{g}_{0}}=\frac{\partial \ln G\left(x, t_{0}\right)}{\partial \ln x^{-1}} .
$$

In these equations $t_{0}=\ln \left(Q_{0}^{2} / \Lambda^{2}\right)$ is the starting scale, where $Q_{0}^{2}$ corresponds to the square of the input momentum and $\Lambda$ is the QCD cutoff parameter. These equations can be determined through $\lambda_{\mathrm{g}}$ and $\lambda_{\mathrm{S}}$ for our aims, respectively [16].

On the other hand, based on the Regge-like behaviour of the gluon distribution and the structure function, Eq. (7) can be solved. Integrating it we have

$$
F_{\mathrm{L}}\left(x, Q^{2}\right)=\eta F_{2}\left(x, Q^{2}\right)+\zeta G\left(x, Q^{2}\right),
$$

where

$$
\eta=\frac{4 \alpha_{s}}{3 \pi} \frac{1-x^{2+\lambda_{\mathrm{S}}}}{\left(2+\lambda_{\mathrm{S}}\right)}
$$

and

$$
\zeta=\frac{20 \alpha_{s}}{9 \pi} \frac{\left(2+\lambda_{\mathrm{g}}\right) x^{3+\lambda_{\mathrm{g}}}-\left(3+\lambda_{\mathrm{g}}\right) x^{2+\lambda_{\mathrm{g}}}+1}{\left(2+\lambda_{\mathrm{g}}\right)\left(3+\lambda_{\mathrm{g}}\right)} .
$$

Substituting Eqs. (12) and (13) into Eq. (18) and then into Eq. (2) for each constant value of $x$ gives us a relation of the reduced cross-section $\sigma_{\mathrm{r}}$ with $\lambda_{\mathrm{S}}$ and $\lambda_{\mathrm{g}}$ exponents. On this basis we find that

$$
\begin{aligned}
\sigma_{\mathrm{r}}\left(x, Q^{2}\right)= & F_{2}\left(x, Q_{0}^{2}\right)\left(\frac{Q_{0}^{2}}{Q^{2}}\right)^{b_{\mathrm{s}} \ln x}\left(1-\frac{y^{2}}{Y_{+}} \eta\right) \\
& -\frac{y^{2}}{Y_{+}} \zeta G\left(x, Q_{0}^{2}\right)\left(\frac{Q_{0}^{2}}{Q^{2}}\right)^{b_{\mathrm{g}} \ln x},
\end{aligned}
$$

where $F_{2}\left(x, Q_{0}^{2}\right)$ and $G\left(x, Q_{0}^{2}\right)$ are the input structure function and gluon distribution function. In order to estimate its derivatives, we take the derivatives of Eq.(21) with respect to $\ln y$ for each value of constant $Q^{2}$ and $x$, (i. e. $\left(\mathrm{d} \sigma_{\mathrm{r}} / \mathrm{d} \ln y\right)_{Q^{2}=\text { constant }}$ and $\left.\left(\mathrm{d} \sigma_{\mathrm{r}} / \mathrm{d} \ln y\right)_{x=\text { constant }}\right)$. So we obtain the following results:

$$
\left.\frac{\mathrm{d} \sigma_{r}}{\mathrm{~d} \ln y}\right|_{Q^{2}=\text { const }}=
$$

$$
\begin{aligned}
& -\frac{\partial F_{2}\left(x, Q_{0}^{2}\right)}{\partial \ln x}\left(\frac{Q_{0}^{2}}{Q^{2}}\right)^{b_{\mathrm{s}} \ln x}\left(1-\eta \frac{y^{2}}{Y_{+}}\right) \\
& -F_{2}\left(x, Q_{0}^{2}\right)\left(\frac{Q_{0}^{2}}{Q^{2}}\right)^{b_{\mathrm{s}} \ln x} \frac{\lambda_{\mathrm{S}}}{\ln \left(Q^{2} / \Lambda^{2}\right)} \ln \frac{Q_{0}^{2}}{Q^{2}}\left(1-\eta \frac{y^{2}}{Y_{+}}\right) \\
& -F_{2}\left(x, Q_{0}^{2}\right)\left(\frac{Q_{0}^{2}}{Q^{2}}\right)^{b_{\mathrm{s}} \ln x}\left(2 y^{2} \frac{2-y}{Y_{+}^{2}} \eta+\frac{y^{2}}{Y_{+}} \frac{4 \alpha_{s}}{3 \pi} x^{2+\lambda_{\mathrm{S}}}\right) \\
& +\frac{\partial G\left(x, Q_{0}^{2}\right)}{\partial \ln x}\left(\frac{Q_{0}^{2}}{Q^{2}}\right)^{b_{\mathrm{g}} \ln x} \frac{y^{2}}{Y_{+}} \zeta \\
& +G\left(x, Q_{0}^{2}\right)\left(\frac{Q_{0}^{2}}{Q^{2}}\right)^{b_{\mathrm{g}} \ln x} \frac{\lambda_{\mathrm{g}}}{\ln \left(Q^{2} / \Lambda^{2}\right)} \ln \frac{Q_{0}^{2}}{Q^{2}} \frac{y^{2}}{Y_{+}} \zeta \\
& -G\left(x, Q_{0}^{2}\right)\left(\frac{Q_{0}^{2}}{Q^{2}}\right)^{b_{\mathrm{g}} \ln x} \\
& \quad \times\left[2 y^{2} \frac{2-y}{Y_{+}^{2}} \zeta-\frac{y^{2}}{Y_{+}} \frac{20 \alpha_{s}}{9 \pi}\left(x^{3+\lambda_{\mathrm{g}}}-x^{2+\lambda_{\mathrm{g}}}\right)\right]
\end{aligned}
$$

and

$$
\begin{aligned}
& \left.\frac{\mathrm{d} \sigma_{r}}{\mathrm{~d} \ln y}\right|_{x=\text { const }}= \\
& -F_{2}\left(x, Q_{0}^{2}\right)\left(\frac{Q_{0}^{2}}{Q^{2}}\right)^{b_{\mathrm{S}} \ln x}\left\{\lambda_{\mathrm{S}} \frac{\ln x}{\ln \left(Q^{2} / \Lambda^{2}\right)}\left(1-\frac{y^{2}}{Y_{+}} \eta\right)\right. \\
& +2 y^{2} \frac{2-y}{Y_{+}^{2}} \eta-\frac{y^{2}}{Y_{+}} \frac{4 \alpha_{s}}{3 \pi}\left[\frac{1-x^{2+\lambda_{\mathrm{S}}}}{\left(2+\lambda_{\mathrm{S}}\right) \ln \left(Q^{2} / \Lambda^{2}\right)}\right. \\
& \left.\left.+\frac{\lambda_{\mathrm{S}} \ln x x^{2+\lambda_{\mathrm{S}}}}{\left(2+\lambda_{\mathrm{S}}\right) \ln \left(Q^{2} / \Lambda^{2}\right)}+\frac{\lambda_{\mathrm{S}}\left(1-x^{2+\lambda_{\mathrm{s}}}\right)}{\left(2+\lambda_{\mathrm{S}}\right)^{2} \ln \left(Q^{2} / \Lambda^{2}\right)}\right]\right\} \\
& +G\left(x, Q_{0}^{2}\right)\left(\frac{Q_{0}^{2}}{Q^{2}}\right)^{b_{\mathrm{g}} \ln x}\left\{\lambda_{\mathrm{g}} \frac{\ln x}{\ln \left(Q^{2} / \Lambda^{2}\right)} \frac{y^{2}}{Y_{+}} \zeta\right. \\
& -2 y^{2} \frac{2-y}{Y_{+}^{2}} \zeta-\frac{y^{2}}{Y_{+}} \frac{20 \alpha_{s}}{9 \pi}\left[\left(-2 x^{3+\lambda_{\mathrm{g}}}+3 x^{2+\lambda_{\mathrm{g}}}-1\right.\right. \\
& \left.+\left(2+\lambda_{\mathrm{g}}\right) x^{3+\lambda_{\mathrm{g}}} \lambda_{\mathrm{g}} \ln x-\left(3+\lambda_{\mathrm{g}}\right) x^{2+\lambda_{\mathrm{g}}} \lambda_{\mathrm{g}} \ln x\right) \\
& \quad \times\left[\left(2+\lambda_{\mathrm{g}}\right)\left(3+\lambda_{\mathrm{g}}\right) \ln \left(Q^{2} / \Lambda^{2}\right)\right]^{-1} \\
& \quad-\frac{\left(2+\lambda_{\mathrm{g}}\right) x^{3+\lambda_{\mathrm{g}}}-\left(3+\lambda_{\mathrm{g}}\right) x^{2+\lambda_{\mathrm{g}}}+1}{\left(2+\lambda_{\mathrm{g}}\right)^{2}\left(3+\lambda_{\mathrm{g}}\right) \ln \left(Q^{2} / \Lambda^{2}\right)} \lambda_{\mathrm{g}}-
\end{aligned}
$$




$$
\left.\left.-\frac{\left(2+\lambda_{\mathrm{g}}\right) x^{3+\lambda_{\mathrm{g}}}-\left(3+\lambda_{\mathrm{g}}\right) x^{2+\lambda_{\mathrm{g}}}+1}{\left(2+\lambda_{\mathrm{g}}\right)\left(3+\lambda_{\mathrm{g}}\right)^{2} \ln \left(Q^{2} / \Lambda^{2}\right)} \lambda_{\mathrm{g}}\right]\right\} .
$$

Equations (21)-(23) are our main results. Therefore the reduced cross-section and its derivatives with respect to $\ln y$ at $x$ or $Q^{2}$ constant based on $\lambda_{\mathrm{S}}$ and $\lambda_{\mathrm{g}}$ exponents are determined.

\section{Calculation and results}

In this paper we employed the Regge-like behaviour of structure function and gluon distribution to calculate the reduced cross-section and its derivatives with respect to $\ln y$ at $Q^{2}=20 \mathrm{GeV}^{2}$ and $Q^{2}=22.5 \mathrm{GeV}^{2}$. We have taken the exponents $\lambda_{\mathrm{S}}$ and $\lambda_{\mathrm{g}}$ for our calculation from Ref. [16]. In these calculations $\lambda_{\mathrm{S}}=0.270$ and $\lambda_{\mathrm{g}}=0.370$ at $Q^{2}=20 \mathrm{GeV}^{2}$, also $\lambda_{\mathrm{S}}=0.274$ and $\lambda_{\mathrm{g}}=0.372$ at $Q^{2}=22.5 \mathrm{GeV}^{2}$, respectively. The same analysis can be done for other $Q^{2}$ values, too. In our calculations, the value of $\Lambda$ is as used in Ref. [13], i. e., $\Lambda=$ $292 \mathrm{MeV}$. We have taken the parameters of the input distributions $x q(x)=a_{q} x^{b_{q}}(1-x)^{c_{q}}\left[1+d_{q} \sqrt{x}+e_{q} x\right]$ for $x g\left(x, Q_{0}^{2}\right), V\left(x, Q_{0}^{2}\right)$, and $A\left(x, Q_{0}^{2}\right)$ at the initial scale $Q_{0}^{2}=4 \mathrm{GeV}^{2}$ using $\mathrm{H} 1$ and BCDMS data for $Q^{2} \gg 3.5 \mathrm{GeV}^{2}$ [17]. The parameterisations used are summarized in Table 1. The initial proton structure

Table 1. Parameters of the input distributions for $x g\left(x, Q^{2}\right)$,

$$
V\left(x, Q^{2}\right) \text {, and } A\left(x, Q^{2}\right) .
$$

\begin{tabular}{cccccc}
\hline & $a$ & $b$ & $c$ & $d$ & $e$ \\
\hline gluon & 1.10 & -0.247 & 17.5 & -4.83 & 68.2 \\
$V$ & 86.3 & 1.47 & 4.48 & -2.12 & 1.60 \\
$A$ & 0.229 & -0.130 & 19.7 & -3.82 & 29.8 \\
\hline
\end{tabular}

function $F_{2}\left(x, Q_{0}^{2}\right)$ is obtained from the decomposition of the structure function into two independent combinations of parton distribution functions $V\left(x, Q^{2}\right)$ and $A\left(x, Q^{2}\right)$, according to

$$
F_{2}=\frac{1}{3} x V+\frac{11}{9} x A \text {. }
$$

The results of the calculations are shown in Figs. 13. In Fig. 1 a comparison is made between our obtained values for the reduced cross-section and the H1 Collaboration [17] data, indicating the fact that the reduced cross-section $\sigma_{\mathrm{r}}$ can be determined with reasonable precision. As can be seen, there is some rate of increment as observed in the $\mathrm{H} 1$ data, but with a somewhat smaller rate. For $Q^{2}$ constant, there is a crossover point for both of the curves whose prediction is numerically

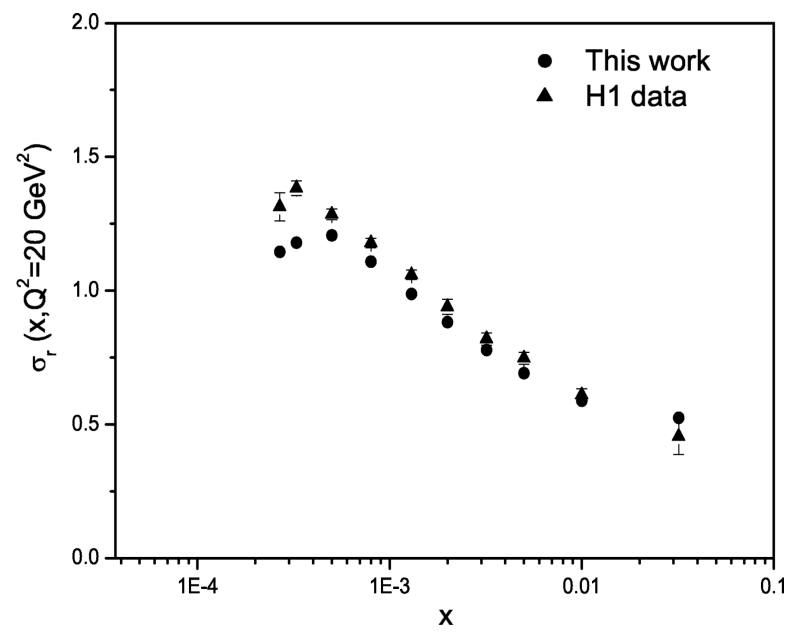

Fig. 1. Determination of the reduced DIS scattering cross-section (closed points). Triangles represent data from the H1 Collaboration [17] with the total errors including the experimental and model uncertainly of the QCD fit.

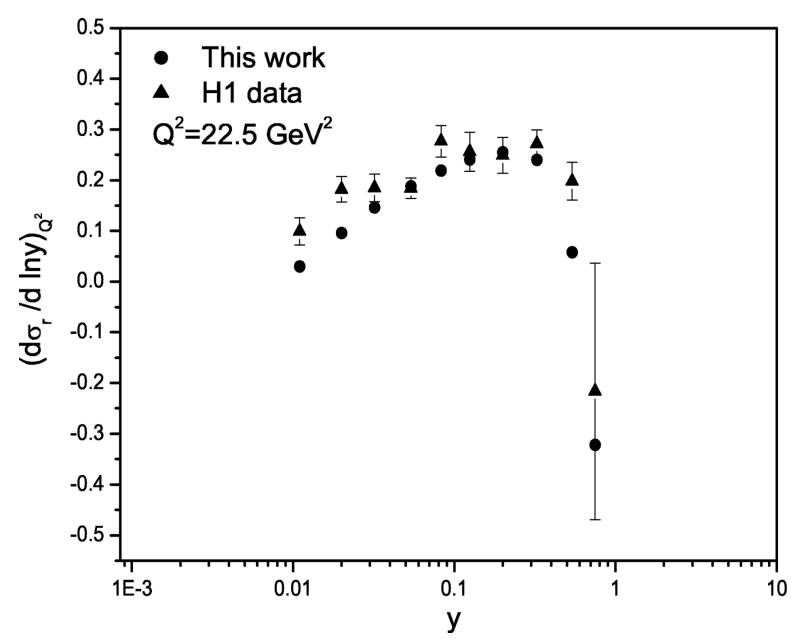

Fig. 2. Determination of the derivative $\mathrm{d} \sigma_{\mathrm{r}} / \mathrm{d} \ln y=-\mathrm{d} \sigma_{\mathrm{r}} / \mathrm{d} \ln x$ at fixed $Q^{2}$ (closed points). Triangles represent data from the H1 Collaboration [17] with the total error.

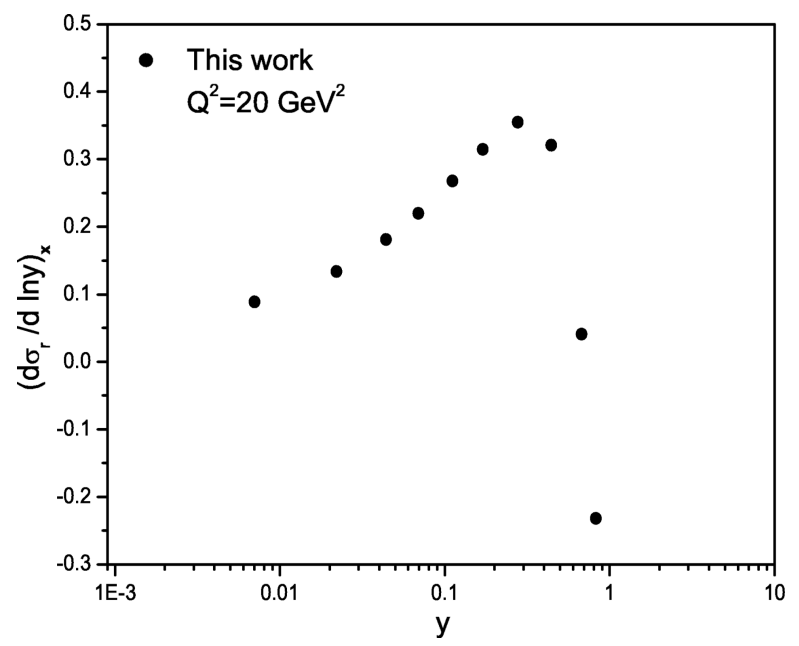

Fig. 3. Determination of the derivative $\mathrm{d} \sigma_{\mathrm{r}} / \mathrm{d} \ln y=+\mathrm{d} \sigma_{\mathrm{r}} / \mathrm{d} \ln Q^{2}$ at fixed $x$ (closed points). 
equal. The resulting $y$-derivatives of $\sigma_{\mathrm{r}}$ with respect to $\ln y$ at fixed $Q^{2}$ are shown in comparison with those extracted from H1 data [17] in Fig. 2. The error bars in the H1 data [17] include the total errors. Finally, the data in Fig. 3 show derivative of the reduced cross-section with respect to $\ln y$ at $x$ constant. We see that there is good agreement between the two data sets, leading us to conclude that we can obtain the reduced cross-section and its derivative based on the gluon and structure function exponents at all $y$ values. The increase rates are almost the same for all $Q^{2}$ values but at low $x$ we see its increment rate is decreased. We expect that NLO QCD calculations of the longitudinal structure function are more correct and its solutions will give a better fit to global data and parameterizations at very low $x$.

In conclusion, based upon the Regge-like behaviour of the gluon and the structure function at low $x$, an approximate method for the calculation $\sigma_{\mathrm{r}}$ and its derivatives with respect to $\ln y$ is presented. In this method $\sigma_{\mathrm{r}}$ and its derivatives with respect to $\ln y$ for low $x$ values at $Q^{2}$ constant using the DGLAP evolution equation without knowledge of the longitudinal structure function $F_{\mathrm{L}}\left(x, Q^{2}\right)$ are determined. Careful investigation of our results show a good agreement with the previous published data based on an "extrapolation method" and a "derivative method". There is however a region, a $Q^{2}$ interval, where the two regimes, Regge and perturbative QCD, are compatible. We have seen that we can use a Regge-like theory which constrains the initial parton densities at $Q^{2}=Q_{0}^{2}$ and obtain the distributions at higher virtualities with the DGLAP evolution equation. These comparisons indicate that the forms of obtained reduced cross-section and its derivatives are similar to those predicted from experimental data. More corrections with NLO QCD calculations will be needed to refine this observation at high $y$. To summarize, we find that the model described in Eqs. (21)-(23), together with the DGLAP evolution, provides a simple and economic solution that could be useful for further practical applications, for example in nuclear physics. In addition, we can conclude that the initial input and its evolution give a good fit to the experimental data in range of the variables $x$ and $Q^{2}$.

\section{References}

[1] R.G. Roberts, The Structure of the Proton (Cambridge University Press, Cambridge, 1990).
[2] N. Gogitidze, Determination of the longitudinal structure function $F_{L}$ at HERA, J. Phys. G 28, 751 (2002).

[3] N. Ghahramany and G.R. Boroun, Extraction of the structure function $F_{2}\left(x, Q^{2}\right)$ at low $x$ from the crosssection derivative, Phys. Lett. B 528, 239 (2002).

[4] A.M. Cooper-Sarkar, R.C.E. Devenish, and A. De Roeck, Structure functions of the nucleon and their interpretation, Int. J. Mod. Phys. A 113, 3385 (1998).

[5] A.W. Thomas and W. Weise, The Structure of the Nucleon (Wiley-VCH, Berlin, 2001).

[6] P.D. Collins, An Introduction to Regge Theory and High-Energy Physics (Cambridge University Press, Cambridge, 1997).

[7] A. Capella et al., Structure function and low $x$, Phys. Lett. B 337, 358 (1994).

[8] L. Csernai et al., From Regge behavior to DGLAP evolution, Eur. Phys. J. C 24, 205 (2002).

[9] Yu.L. Dokshitzer, Sov. Phys. JETP 46, 641 (1977); G. Altarelli and G. Parisi, Nucl. Phys. B 126, 298 (1977); V.N. Gribov and L.N. Lipatov, Sov. J. Nucl. Phys. 15, 438 (1972).

[10] P. Desgrolard, A. Lengyel, and E. Martynov, Pomeron effective intercept-logarithmic derivatives of $F_{2}\left(x, Q^{2}\right)$ in DIS and Regge models, J. High Energy Phys. 02, 029 (2002).

[11] J. Gayler, on behalf of the H1 Collaboration, The rise of the proton structure function $F_{2}$ towards low $x$, Acta Phys. Pol. B 33, 2841 (2002).

[12] G. Soyez, Global QCD fit from $Q^{2}=0$ to $Q^{2}=$ $30000 \mathrm{GeV}^{2}$ with Regge compatible initial condition, Phys. Rev. D 71, 076001 (2005); C. Lopez et al., Z. Phys. C 72, 561 (1996).

[13] The H1 Collaboration, C. Adloff et al., On the rise of the proton structure function $F_{2}$ towards low $x$, Phys. Lett. B 520, 183 (2001).

[14] A.M. Cooper-Sarkar and R.C.E. Devenish, The rise and fall of $F_{2}$ at low $x$, Acta Phys. Pol. B 34, 2911 (2003).

[15] R.K. Ellis, Z. Kunszt, and E.M. Levin, The evolution of parton distributions at small $x$, Nucl. Phys. B 420, 517 (1994); R.K. Ellis, W.J. Stirling, and B.R. Webber, QCD and Collider Physics (Cambridge University Press, Cambridge, 1996).

[16] G.R. Boroun and B. Rezaie, Approximate method for calculating the exponent of the gluon distribution, $\lambda_{g}$, and the exponent of the structure function, $\lambda_{S}$, at low $x$, Phys. At. Nucl. 71, 1077 (2008).

[17] The H1 Collaboration, C. Adloff et al., Deep inelastic inclusive $e p$ scattering at low $x$ and a determination of $\alpha_{s}$, Eur. Phys. J. C 21, 33 (2001). 


\title{
REDUKUOTOJO SKERSPJŪVIO IR JO IŠVESTINIŲ SPRENDINYS NEDIDELE்MS $x$ VERTĖMS, REMIANTIS GLIUONŲ IR SANDAROS FUNKCIJŲ EKSPONENTĖMIS
}

\author{
G.R. Boroun
}

Razi universitetas, Kermanšachas, Iranas

\section{Santrauka}

Pateiktos analizinès redukuotojo skerspjūvio ir jo išvestinių išraikos $\ln y$ atžvilgiu, kai Bjorkeno parametras $x$ yra mažas. Išspręstos DGLAP evoliucinès lygtys singletinei ir gliuonų sandaros funkcijai, grindžiamos Redže tipo gliuonu pasiskirstymo ir sanda- ros funkcijos elgsena šioje riboje. Skaitmeniškai gauti rezultatai palyginti su HERA bandymo H1 duomenimis, kai $x$ maži. Visus rezultatus galima nuosekliai aprašyti naudojant perturbacinę kvantinę chromodinamiką. 